\title{
Pathological findings in homocystinuria
}

\author{
J. B. GIBSON ${ }^{1}$, NINA A. J. CARSON, AND D. W. NEILL ${ }^{2}$ \\ From the Departments of Pathology and of Child Health, and the \\ Biochemistry Laboratory, The Queen's University of Belfast, and \\ Royal Belfast Hospital for Sick Children, and Royal Victoria Hospital, Belfast
}

SYNOPSIS Pathological findings are described in four cases of a new aminoaciduria in which homocystine is excreted in the urine. All the patients were mentally retarded children. Three of them presented diagnostic features of Marfan's syndrome. Necropsy on one case and biopsy findings in the others are described. Fatty change occurs in the liver. The most striking lesions are vascular. Metachromatic medial degeneration of the aorta and of the elastic arteries in the necropsied case are considered in relation to Marfan's syndrome. Other changes, particularly thrombosis which is prevalent in homocystinuria, suggest the possibility of a platelet defect. The findings are discussed in respect of an upset in the metabolism of sulphur-containing amino-acids and with particular reference to Marfan's syndrome.

A systematic search for metabolic abnormalities in mentally retarded individuals in Northern Ireland revealed a hitherto unrecognized specific aminoaciduria, in which the sulphur-containing aminoacid homocystine is excreted (Carson and Neill, 1962).

The amino-aciduria has been detected in seven males and three females from seven families. Two siblings are affected in each of three unrelated families (Carson, 1963). The pattern of inheritance appears to be that of an autosomal recessive with normal parents. The patients ranged from $1 \frac{1}{2}$ to 32 years of age on detection. Many of the clinical features were remarkably constant. All the patients were mentally retarded, the highest I.Q. being 53 at the time of diagnosis. With one exception they were fair haired and fair complexioned. A high malar flush and ectopia lentis were regularly present. The patients walked with a rather high-stepping spastic gait and pes cavus and genu valgum were noted in all.

The clinical findings will be reported in detail elsewhere and the biochemical aspects have been investigated by Carson, Cusworth, Dent, Field, Neill, and Westall (1963).

The pathological findings have not been described previously. In addition to ectopia lentis, three of the 10 patients originally diagnosed showed kyphoscoliosis and chest deformity with arachnodactyly.

1 Present address: Department of Pathology, the University of Hong Kong

${ }^{2}$ Reprints will be available from D. W. Neill. Royal Victoria Hos pital, Belfast.

Received for publication 29 November 1963.
Their appearance was that of Marfan's syndrome and in fact two of them had been designated previously as classical examples of Marfan's syndrome in published studies of that condition (case A4, Lynas, 1958; case 4, Loughridge, 1959). In another of the older patients kyphoscoliosis and chest deformity were marked but true arachnodactyly was not present. The striking association between homocystinuria and Marfan's syndrome is explored in this study of the morbid anatomical findings.

Necropsy findings are reported in one of the 10 cases of homocystinuria which did not present all the diagnostic features of Marfan's syndrome in life (case 1). Operations on two of the cases with overt Marfan's syndrome (cases 2 and 3), a nephrectomy and liver biopsy, provided further material for this report. A needle biopsy of the liver from a fourth case is also recorded.

\section{CASE 1}

A boy, J.R., died at Muckamore Abbey Special Care Hospital on 5 January 1963 at the age of 6 years 11 months.

FAMILY HISTORY The parents are healthy; both are a little below average in stature; homocystinuria, ectopia lentis, and arachnodactyly are absent; there is no consanguinity. Four younger brothers and one younger sister of J.R. are alive and of average intelligence; there is no homocystinuria, ectopia lentis, or arachnodactyly in these children; one of the boys is under observation for steatorrhoea but they are healthy otherwise. One miscarriage occurred in the family and the youngest member 
died at 3 days of age with multiple congenital abnormalities. S.R., an elder brother of the patients, now aged 9 years, is mentally defective; he developed swelling of the abdomen and of the legs at 22 months and venous thrombosis was diagnosed; this boy has ectopia lentis but no arachnodactyly: homocystinuria was detected at the age of 7 years. The urinary chromatogram was normal in other respects.

HISTORY J.R. was considered normal at birth and until the age of 13 months, but a radiograph of the carpus then showed only one carpal centre of ossification instead of the normal two. At this time there was an episode of vomiting; convulsions and coma occurred and a permanent right-sided hemiparesis followed. Diffuse cerebral atrophy was demonstrated by air encephalography and dural thrombophlebitis was diagnosed.

He was admitted to Muckamore Abbey Hospital in April 1961, at the age of 5 years as a person requiring special care. His mental age was about 2 years. He was able to walk unaided with difficulty and was reluctant to stand on his right leg. The fingers were short and stubby. He was stated to be sensitive to sunlight and had a malar flush. During his two years in hospital, a few epileptiform attacks occurred and he received phenobarbitone, $1 \frac{1}{2}$ grains per day. Ectopia lentis was observed and homocystine was detected in the urine by routine paper chromatography on two occasions. In September 1962 an inflammation of the right gastrocnemius was treated successfully with penicillin but he could no longer walk without assistance. At the end of December, attacks of vomiting and a few grand mal and Jacksonian seizures occurred, followed by pyrexia, and he died on 5 January 1963.

\section{FINDINGS AT NECROPSY ON CASE 1}

The body is that of a remarkably fair-haired child of about the normal height for his age. The eyes are rather sunken, the neck long, and the skull assymetrical on account of a little bulging on the right posteriorly. There are no external skull bosses. There are several broken and carious teeth of normal colour. The body is poorly covered with fat and the lower limbs, particularly the right, are wasted. The thighs curve forward a little because of bony deformity and there is genu valgum. The hands are rather spade-like and have two transverse palmar creases. The fingers are not spatulate or elongated. The testes are in the scrotum.

There is no bony abnormality of the chest or vertebral column. The lungs (right $125 \mathrm{~g}$., left $120 \mathrm{~g}$.) are slightly collapsed. The pulmonary artery is not dilated; it shows slight transverse intimal ridging. The heart $(100 \mathrm{~g}$.) is well formed and its chambers are in proportion. Some small shock haemorrhages are seen in the left ventricular endocardium and no other myocardial lesion. The endocardium of the left atrium and the mitral valve cusps are slightly

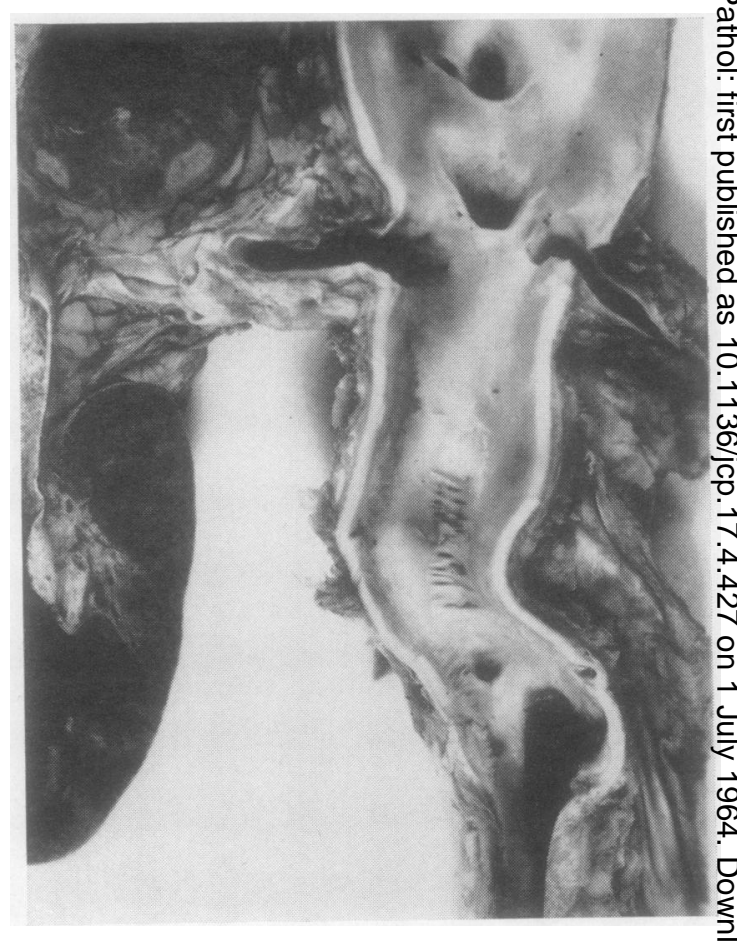

FIG. 1. Case 1. Lower aorta opened posteriorly: the left kidney is infarcted and thrombus is seen in the lefo renal artery and at the aortic bifurcation. There is trans verse striation of the anterior aortic wall above the inferio $\overrightarrow{5}$ mesenteric artery. The aorta is thickened round the mouth of the left renal artery and of the superior mesenteris artery which is dilated.

thickened by fibrous tissue. The tricuspid valve is normal. The pulmonary valve is $4.5 \mathrm{~cm}$. in circum ference and the aortic valve is $5 \mathrm{~cm}$. The coronary ostia lie at the normal level and there are ne aneurysms of the sinuses of Valsalva. The arteries appear normal.

The gastrointestinal tract, pancreas and adrenats are normal.

The liver (500 g.) is mottled and congested. Af area round the ligamentum teres posteriorly is morro fatty than the rest. The vessels in the porta hepatis and in the liver itself are not obstructed. The biliary apparatus is normal and there are no gan stones. The spleen (50 g.) is firmer than usual 略 several poorly defined areas under puckered regiors of the capsule.

The right kidney (60 g. approximately) and i vessels appear normal. The left kidney (80 $\vec{Q}$ approximately) is swollen, haemorrhagic, and almost completely infarcted. The renal artery is totally blocked by mixed thrombus which is softened in places (Fig. 1). There are no old scars of tie 
kidney and there are no renal or other stones. Ureters, bladder, and prostate are normal. The urine is not bloodstained.

The aorta is a little dilated in the ascending part; the arch is moderately dilated and the wall is stretched and thin as far as the left subclavian artery, where the lumen narrows a little and the wall is thickened and encircled by two low band-like elevations of the lining each about $2 \mathrm{~mm}$. broad lying above and below the mouth of the subclavian. Just below it are a few wispy strands and tiny depressions of the intima; the vessel is unduly friable around them. The aorta is again a little dilated here and the dilatation continues down to the level of the renal arteries. In the aortic wall beside the left adrenal there is a narrow line of transverse striations like comb marks extending for a few centimetres. The lower part of the line is covered by laminated mixed thrombus several millimetres thick ending about the level of the coeliac axis. Another area of transverse striation, $1.5 \times 0.5 \mathrm{~cm}$., is seen on the anterior wall of the aorta just above the mouth of the inferior mesenteric artery (Fig. 1). The lowest part of the aorta is occluded by adherent thrombus which is chiefly red and extends into the common iliac arteries.

The mouth of the coeliac axis is patent, but within a few millimetres the lumen is occluded by mixed thrombus which extends into the proximal part of the hepatic artery. The wall of the coeliac artery is thick. The splenic artery wall is very thick and the lumen minute. The mouth of the superior mesenteric artery in the aorta is a little dilated and the vessel walls are thickened there (Fig. 1). Immediately distal the artery is dilated to three times its normal diameter in a fusiform aneurysm about $2 \mathrm{~cm}$. long. Mixed thrombus in the left renal artery extends from the kidney to the aorta where the vessel walls are thick and fibrous. The mouth of the right renal artery is also thick walled. The artery is not occluded. The common iliac arteries have thick fibrous walls. There is no thrombus or other lesion of the superior or inferior venae cavae.

The thyroid and other structures of the neck appear normal.

The middle of the left femur is bowed forwards and the lower end of the shaft deviates medially, in keeping with the genu valgum. The lower end of the bone is a little enlarged and the epiphyseal line is not straight but rises in the middle. The centre of ossification of the greater trochanter (normally appearing at 5 years) is $3 \mathrm{~mm}$. in diameter and there is no centre for the lesser trochanter (normally appearing at 10 years). The marrow is red in the upper half of the bone.

The deformed outlines of the skull follow the

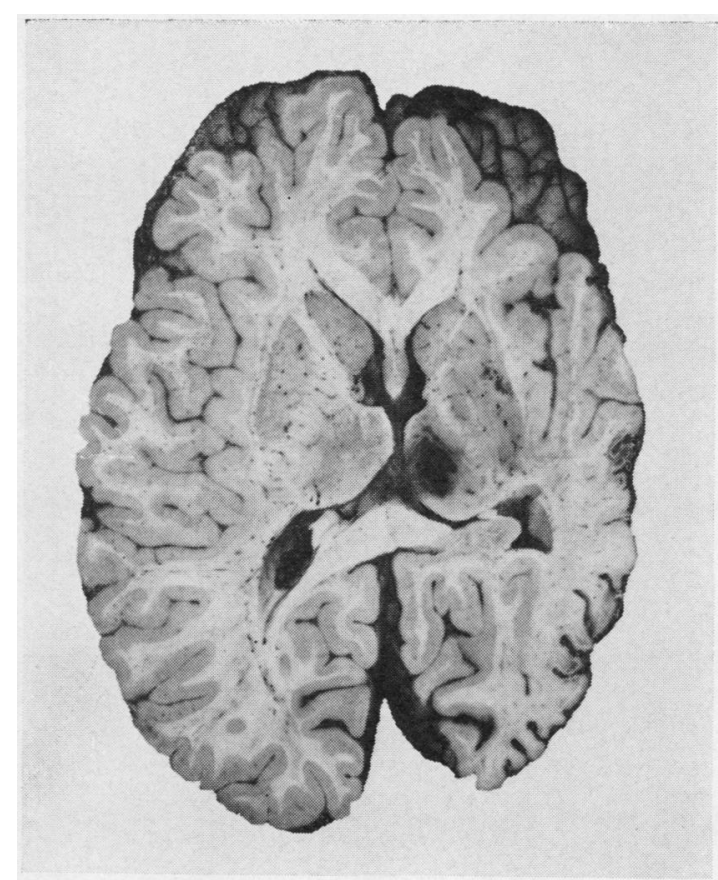

FIG. 2. Case 1. Horizontal section of brain: healed venous infarcts of left occipital cortex and thalamus.

contours of the underlying brain. A bony abnormality unconnected with this deformity is seen in the posterior fossa where the basion bulges upward a little. Just lateral to the margins of the foramen magnum two rounded bosses arise from the occipital bone on each side. They are 5 to $7 \mathrm{~mm}$. in diameter and are composed of well-formed bone continuous in texture with that of the skull. These roughly symmetrical domes of bone have not compressed the brain to any discernible degree.

The dura round the foramen magnum and the upper part of the spinal cord is thicker and more fibrous than normal. There is a moderately dense and vascular fibrosis of the dura over the left cerebral hemisphere superolaterally, with fibrous adhesions to the leptomeninges towards the vertex. The superior sagittal sinus is extensively obstructed by recanalized fibrous tissue.

In the brain $(1,250 \mathrm{~g}$.) the left cerebral hemisphere is smaller than the right, particularly in the occipital lobe (Fig. 2). Below the adherent dura, the cortex is brown-tinged and feels a little firmer than normal. The left temporal lobe and the inferior parietal lobule are atrophied posteriorly so that the cortical pattern is small. In the occipital lobe many areas of brownish, healed infarction are disposed in the lower layers of the cortex in a roughly laminar distribution. An orange-brown cystic softening, 


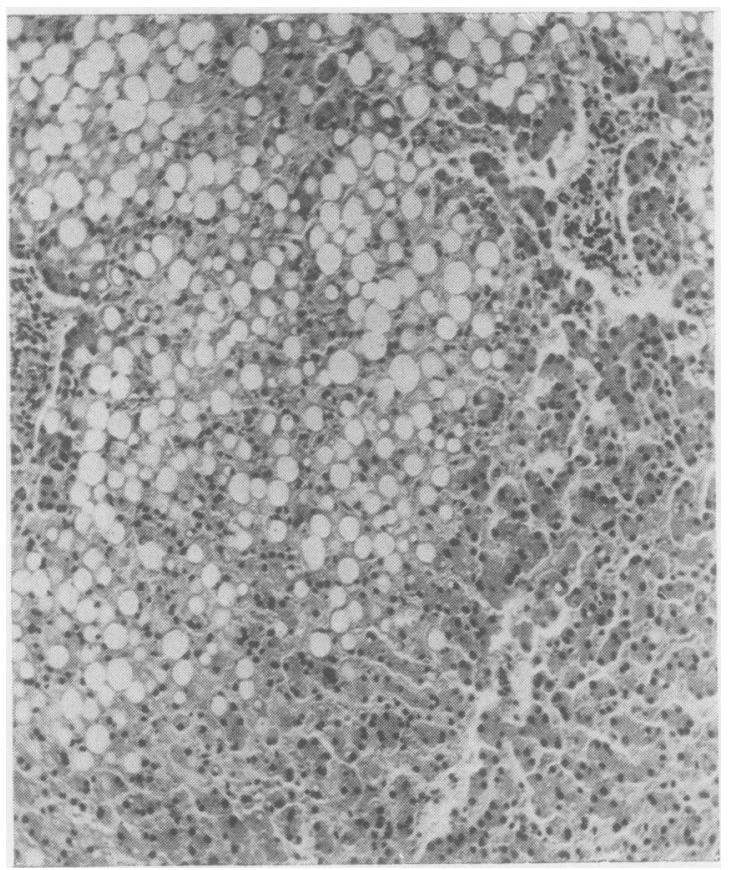

FIG. 3. Case 1. Liver: the edge of an area of fatty change. Haematoxylin and eosin $\times 120$.

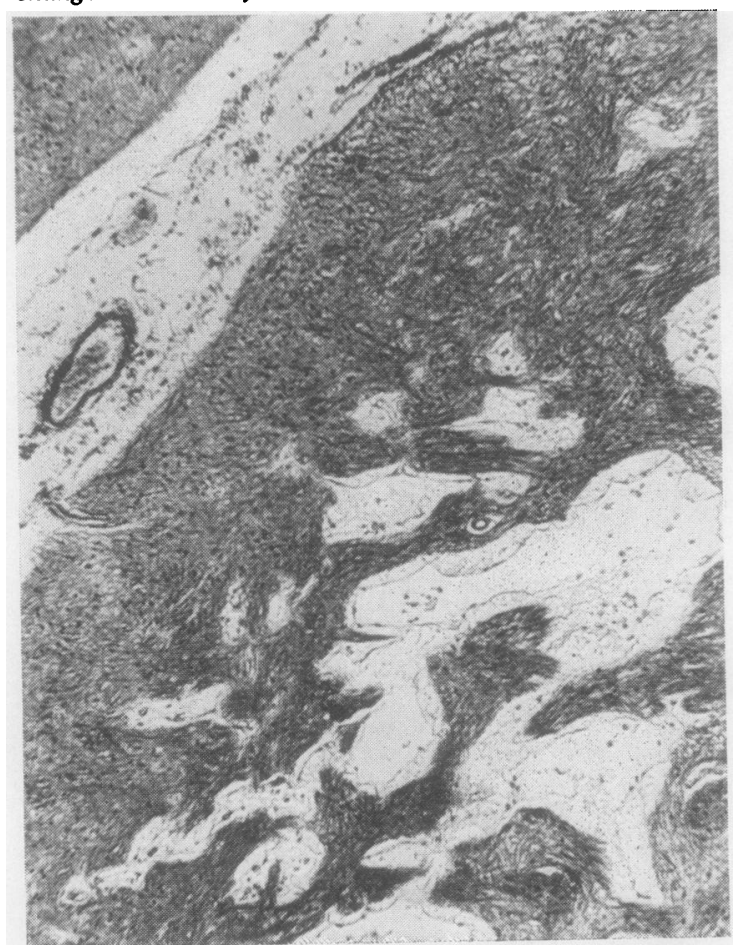

FIG. 4. Case 1. Occipital cortex: healed cystic infarct and fibrous gliosis. Mallory's phosphotungstic-acidhaematoxylin $\times 105$.

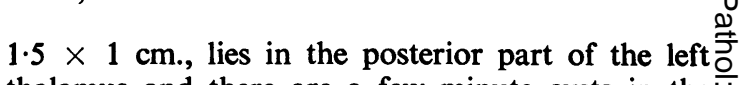
thalamus and there are a few minute cysts in the

The spinal cord shows no lesion. The lepto-o meninges are a little thickened in the cervical region.

The pituitary is not remarkable.

\section{HISTOLOGY IN CASE 1}

Conventional stains on Helly and formalin-fixed. $\vec{\circ}$ sections were supplemented by staining with $1 \% \overrightarrow{\vec{\omega}}$ alcian green 3BX in $1 \%$ glacial acetic acid $(p \mathrm{H} 2)$ of ${ }^{\circ}$ paraffin sections of formalin-fixed blocks postfixed in saturated aqueous mercuric chloride. Meta-:chromasia was detected in formalin-fixed frozen. sections by staining for five minutes with $0.05 \%$ thionine in McIlvaine's buffers at $p \mathrm{H} \mathrm{6,4}$, and 2.5,, $\mathrm{v}$ after prolonged washing in appropriate buffer. Theo $p \mathrm{H} 4$ treatment gave slightly better results than the$p \mathrm{H} 6$; staining at $p \mathrm{H} 2.5$ was inadequate. By this $\varsigma_{\subseteq}$ technique, bright metachromasia was obtained in sites corresponding to those stained with alcian ince paraffin sections. Control material from an infantile case of Marfan's disease gave similar results.

Parenchymatous lesions are not seen in the pancreas, adrenals, lymph nodes, colon, bladder, thymus, thyroid, parathyroid, pituitary, or bonen marrow. The endocardial fibrosis of the left atriumo has no specific features. The fibrous tissue of the aortic valve ring shows diffuse metachromatico staining. The pulmonary valve ring is similarly and less markedly affected.

An early bronchopneumonia is present in the lungs. Interstitial fibrosis, apparently the result of old partial infarction, is seen in the spleen. The livero cells are generally atrophied and some show watery. vacuolation; in the pale areas noted grossly there is fatty infiltration of all except the periportal areas (Fig. 3). The old softenings of the brain are healedg and surrounded by a moderate degree of fibrillarys gliosis (Fig. 4). No specific lesions are detected in sections from other areas of the brain and spinat cord. A slight gliosis and a swelling of the oligodendroglia is recognizable throughout. A few smals nodules of bone are found in fibrosed areas of the dura. In sections of the skull, ribs, femur, and vertebrae abnormal histological features are not seen in the bone.

The chief lesions are vascular. The superiof sagittal sinus and some of its immediate tributariesp are occupied by fully organized and partly ree canalized tissue. The venae cavae are normal. In the left renal vein there is a moderate degree of fibrou endophlebitis. Other veins are normal.

Medium-sized muscular arteries are affected in patchy fashion. Lesions are detected in some of the 


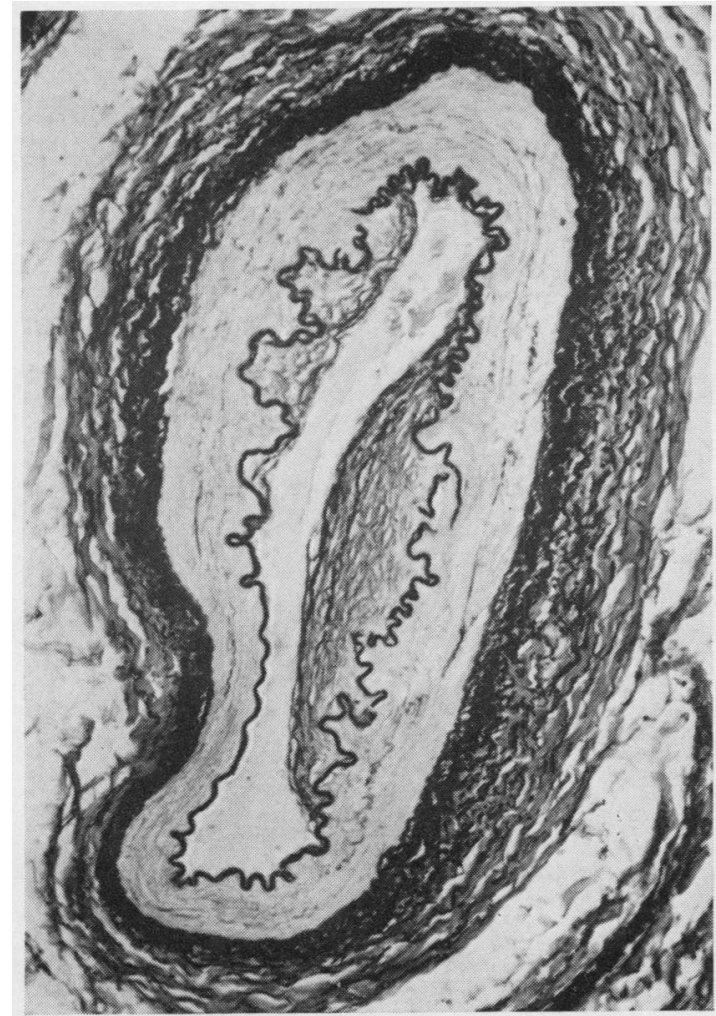

FIG. 5. Case 1. Inferior thyroid artery: elastic fibrils in intimal pads. Prominent and irregular internal elastic lamina. Weigert's elastic tissue stain and Van Gieson $\times 100$.

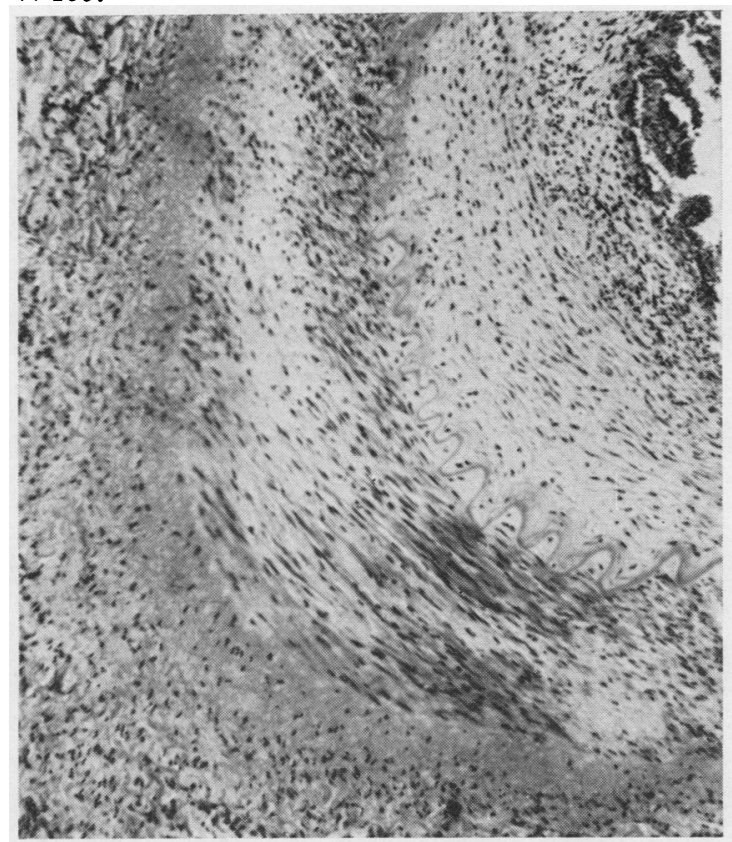

FIG. 6. Case 1. Left renal artery, muscular part: organizing thrombus with intimal fibrosis, swelling of elastica and mucoid degeneration of the media. Haematoxylin and eosin $\times 65$. arteries in the spleen, pancreas. colon, thyroid (Fig. 5), and thymus, pelvis, and left kidney (Fig. 6), with minor or few changes of the same type in arteries of the heart, liver, and right kidney. The internal elastic lamina is prominent and often thick. The main element is intimal fibrosis without deposits of iron pigment or other special features (Fig. 5).- The fibrosis is either symmetrically distributed so as sometimes to cause severe narrowing, as in the splenic artery, or asymmetrically distributed in the form of pads. In the intimal pads, layers of elastic fibrils have split off from the main elastic lamina and small degrees of metachromasia are present. The medial coats of these arteries do not usually stain metachromatically but in some sections of the left renal and splenic arteries the muscle fibres are separated from each other and thinned out by prominent deposits of interstitial material which stains as collagen and also stains metachromatically with moderate intensity (Fig. 6). The external elastic lamina is often swollen. The adventitia is little affected except in vessels such as the proximal part of the hepatic artery where thrombus is being organized; in those sites, the vasa vasorum are congested and there is a little polymorphic infiltration of the media.

Changes of long standing are seen in the proximal parts of the left renal, common iliac, and superior mesenteric arteries and coeliac axis; they are distinct from more recent changes of thrombosis. There is slight concentric intimal fibrosis which is marked in the common iliacs and associated there with some fatty atheromatous plaques. Some areas of intimal fibrosis stain well with alcian. The media is a little less cellular than normal with increased amounts of interstitial material between elastic fibres; the material is metachromatic with toluidine blue and stains with alcian. The elastica is frayed, attenuated or irregularly thickened and disorderly in arrangement in such areas, particularly in the superior mesenteric artery (Fig. 7). A little polymorph infiltration is associated with the aneurysmal dilatation of this vessel. The adventitia is generally not increased; in the left renal artery it is congested and the vascular endothelium is swollen; this may be due to the organization of thrombus in the lumen.

The microscopical structure of the aorta is most altered in the arch above the left subclavian artery where the wall is stretched and thin. The intima of the arch is little altered. The adventitia is congested and the vasa vasorum are prominent; there is a little lymphocytic infiltration and no fibrosis. The media is deeply penetrated by vasa vasorum and slightly fibrosed and the lines of the muscular and elastic fibres are disturbed and disrupted here and there. In little cysts or pools round some of the vasa vasorum 
and in little clumps between cells lie small granular or hyaline areas which are brightly metachromatic. In other places, lines of metachromatic staining extend for $100 \mu$ or more between the fibres. The middle and inner parts of the media are mainly affected.

Similar but less marked metachromatic staining is seen in the ascending and descending parts of the aorta and particularly round the mouths of the renal arteries and neighbouring large branches. Here the findings are complicated by ridges of intimal

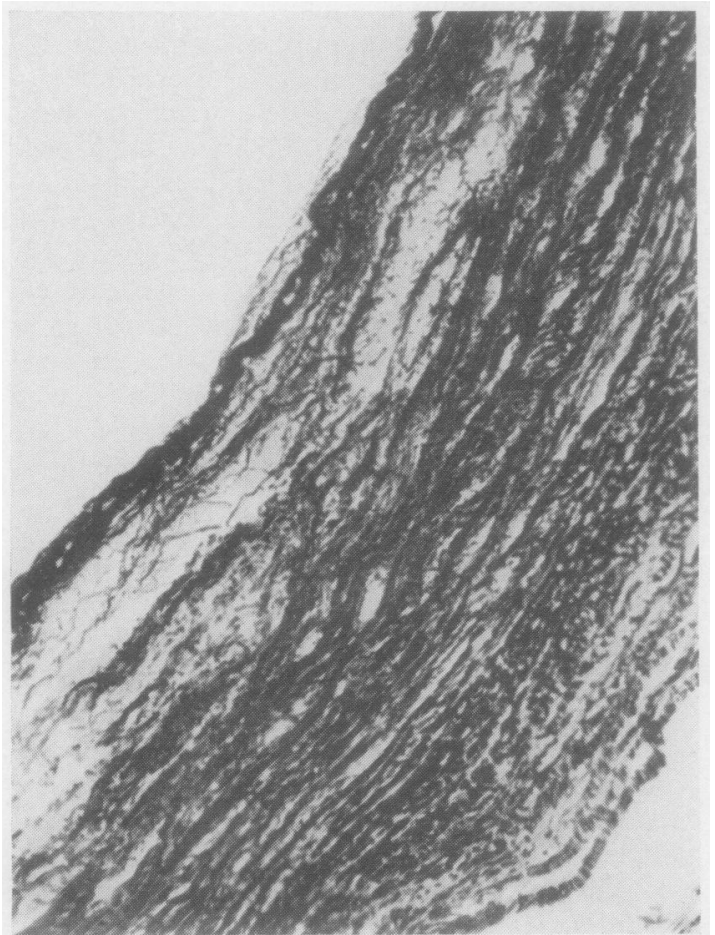

FIG. 7. Case 1. Wall of fusiform aneurysm of superior mesenteric artery: the fibres are stretched and there are defects in the elastica where metachromatic material was present. Weigert's elastic tissue stain and Van Gieson $\times$ 160.

fibrosis. These ridges and striations are composed of moderately cellular fibrous tissue thickly interspersed with elastic fibrils (Figs. 8 and 9). Staining for fibrin and for iron pigment is negative in the ridges and metachromatic staining is generally slight except where the fibrous tissue merges with the process of organization of thrombus in the aortic lumen. The media is not extensively altered but is thinned under the intimal ridges and there the vasa vasorum are prominent and penetrate deeply into the media (Fig. 8). The adventitia is slightly fibrosed and there is a little lymphocytic infiltration round the vasa.

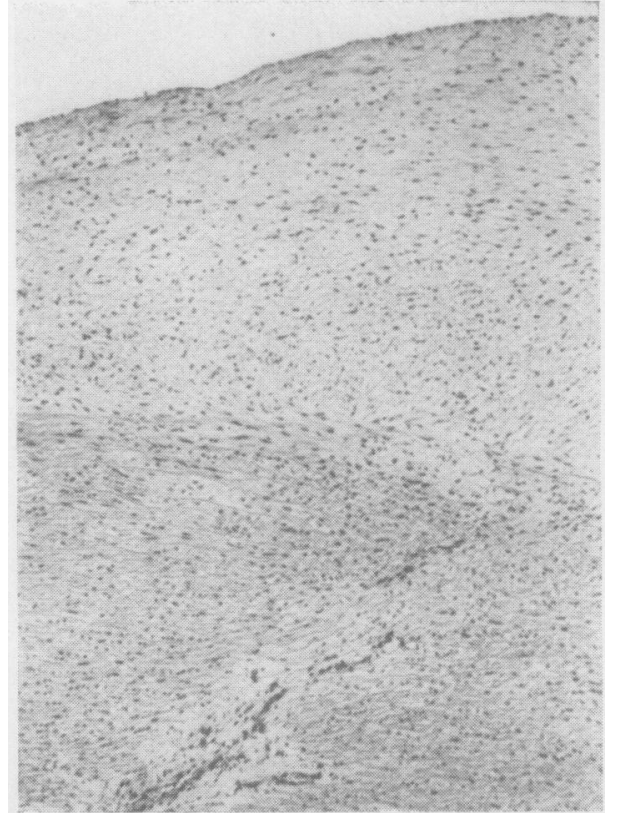

FIG. 8. Case 1. Ridged area of abdominal aorta: $\stackrel{c}{<}$ cellular fibrosis of the intima with a deeply penetrating vas vasis. Haematoxylin and eosin $\times 105$.

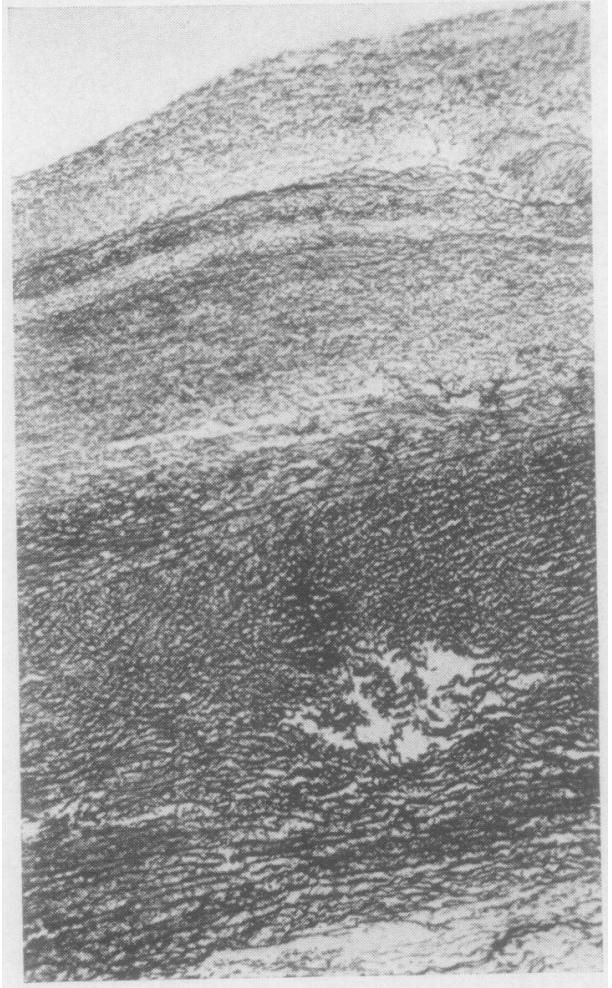

FIG. 9. Case 1. Ridged area of abdominal aorta: elast fibrils have proliferated in the intima. There is a defect the elastic tissue of the media where metachromatif material was present. Weigert's elastic tissue stain ant carmalum $\times 130$. 
The pulmonary artery is of looser texture than normal and between the fibres lie many small deposits of material which stains metachromatically.

\section{CASE 2}

In a boy, G.M., homocystinuria was detected at the age of 13 years; it was the only abnormality found on urinary chromatography. His parents are healthy and unrelated, showing no features of Marfan's syndrome. There were eight children in the family; five are alive and healthy and two died in infancy, one from complications of measles and the other from convulsions. The surviving siblings and the parents have normal urinary amino-acid patterns. A son of a sister of the patient's father was physically similar to the patient; he died at the age of 17 from an unknown cause.

The patient is mentally retarded and his physical appearance is that of Marfan's syndrome (Fig. 10). The extremities are long and thin, the fingers spidery, the chest deformed with kyphoscoliosis, and the palate high and arched; pes cavus, genu valgum, and malar flush are present. Acute glaucoma complicating subluxation of the lens led to enucleation of the left eye in 1958. The ectopic right lens was excised later because of glaucoma.

At the age of 6 years he had an attack of haematuria with oedema of the upper eyelids; blood pressure was $115 / 70 \mathrm{~mm} . \mathrm{Hg}$, blood urea $168 \mathrm{~mm} . \%$. The subsequent

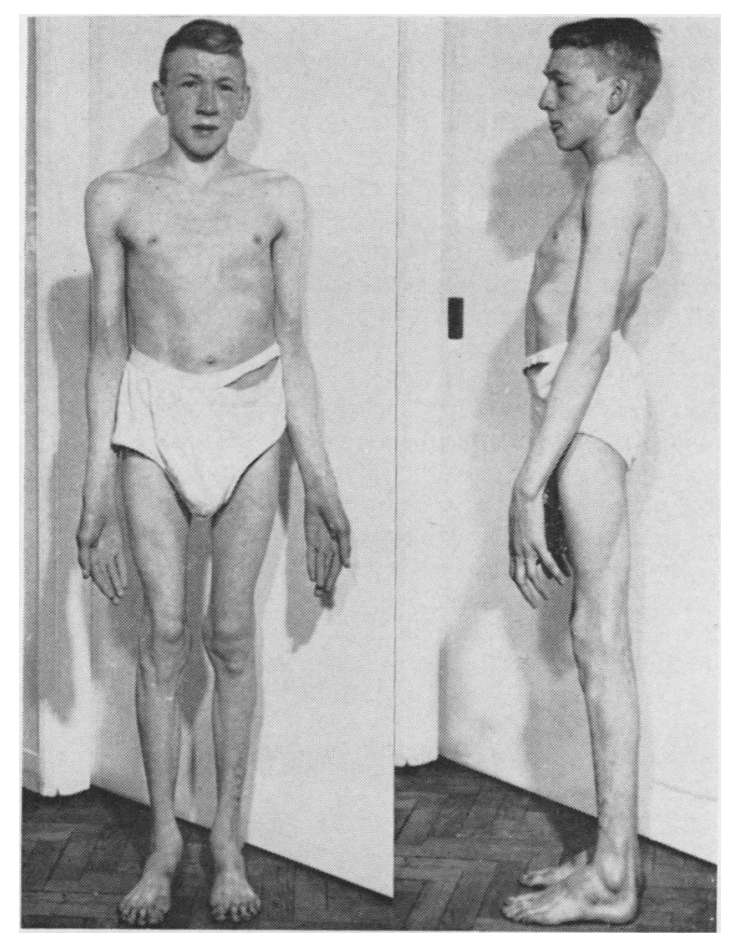

FIG. 10. Case 2, aged 15 years: arachnodactyly, kyphoscoliosis, pes cavus, genu valgum, and other signs of Marfan's syndrome. course of his renal disease and the persistent hypertension that he developed have been described elsewhere (Loughridge, 1959, case 4). In September 1962, then aged 13 years, he was readmitted to hospital; his blood pressure was $170 / 120 \mathrm{~mm} . \mathrm{Hg}$ at the lowest reading. The left kidney, which was ectopic, was removed surgically.

\section{MACROSCOPIC FINDINGS IN CASE 2}

The left kidney, surgically removed, weighed $97 \mathrm{~g}$. It was pale; the capsule was not unduly adherent; the outer surface was slightly granular; the texture was a little firmer than normal. The architectural pattern of cut sections was slightly blurred. There were no marked alterations on naked-eye examination and no infarcts. The main renal artery and some of the interlobular arteries were small in calibre; the walls were symmetrically thickened; others looked normal. Venous lesions were absent. Stones were not present.

\section{HISTOLOGY IN CASE 2}

In histological sections glomerular lesions are not seen and there is nothing to indicate previous glomerulonephritis. The juxtaglomerular apparatus is more prominent than normal. Fibrinoid change has not occurred in glomerular afferrent arterioles; a few are slightly fibrosed. The tubules are well preserved; the medullary interstitial tissue is slightly increased. The pelvis is normal.

The arteries in general have undergone a little symmetrical medial hyperplasia, but irregular asymmetrical proliferation of fibrous and other elements is commoner and more marked, particularly in the vessels of medium calibre (Fig. 11). In the larger arteries, fibrous tissue has proliferated in the intima as a thin irregularly disposed layer and elastic fibres extend into it from the internal elastic lamina; the intima shows marked metachromasia. In the media muscle fibres are often enlarged and somewhat irregularly arranged with an increase of interstitial material which stains as collagen and is, in places, metachromatic; fine elastic fibrils of frayed appearance are scattered through the muscle singly or occasionally together in narrow wedge-shaped segments of the vessels. The external elastic lamina is a little thickened and frayed; there is little or no alteration in the adventitia; sometimes a little metachromasia is seen.

Similar changes have taken place in the arcuate arteries. At this level, intimal fibrosis is often marked in the form of pads and other asymmetrical deposits (Fig. 11). Many elastic fibrils traverse these deposits and the internal elastica is often reduplicated. Sometimes the muscle is irregularly disposed and 


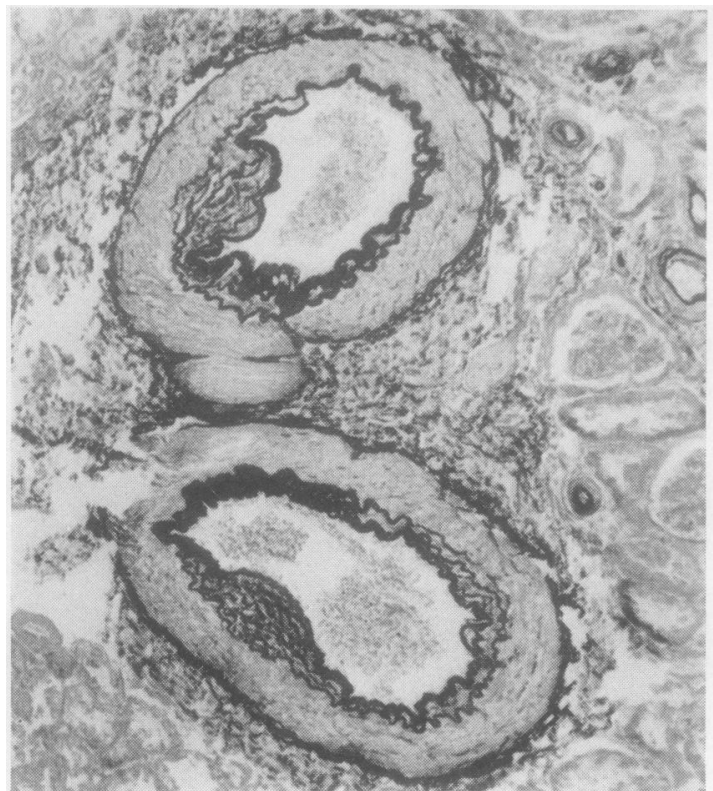

FIG. 11. Case 2. Renal arcuate arteries: asymmetrical fibrous thickenings of the intima with proliferated elastica. Weigert's elastic tissue stain and cermalum $\times 60$.

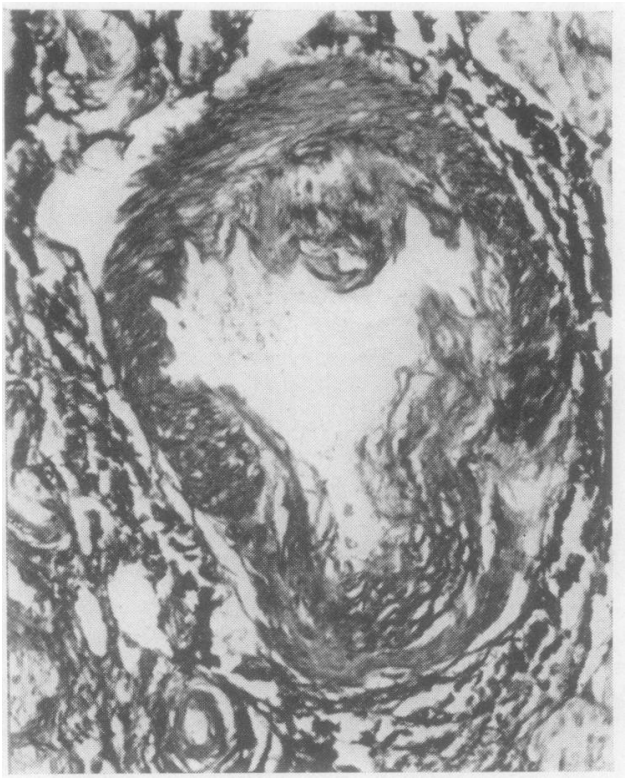

FIG. 12. Case 2. Renal arcuate artery: asymmetrical fibrous intimal pads, irregularly of medial muscle, and segmental fibrosis of media. Picro-Mallory $\times 105$. segmental areas of fibrosis occur in the media (Fig. 12). The walls of the straight arteries are thickened by fibrosis of the media, sometime $\vec{F}$ severely, the internal elastica is often reduplicated ? and there is often a little ring of metachromasio beneath it and hyperplasia of the muscle.

\section{CASE 3}

A boy, A.S., aged 7 years, was admitted to hospita $\vec{\Phi}$ suffering from venous thrombosis in the lower limbs. Several unexplained episodes of venous thrombosis had occurred in the lower limbs since the age of 2 years ans 8 months.

The patient was the illegitimate child of a mother whiे later married another partner. There is no issue of the marriage. The patient was severely mentally retarded an $\&$ was thin with long, slender extremities, hypotonic musculature, fair hair, a high malar flush, bilatera $\Phi$ ectopia lentis, and severe myopia. He walked with much difficulty; kyphoscoliosis, genu valgum, pes cavus, and arachnodactyly were present.

The liver was enlarged and palpable three or four fingerbreadths below the costal margin. Among man investigations, a platelet count of $248,000 / \mathrm{c} . \mathrm{mm}$., $\mathrm{a}$ prothrombin level of $30 \%$, and negative WassermanF and Kahn reactions may be mentioned. The total serun protein was $4.8 \mathrm{~g} . \%$ with a relative increase in globulin Bromsulphalein retention of $23 \%$ at 45 min., serum isocitric dehydrogenase 16.5 units (normal 3.5 to 8 ) psuedo-cholinesterase 16 units (normal 40 to 110 ), zine sulphate flocculation 12 units, were also recorded $\overrightarrow{\vec{D}}$ Bilirubin was normal. A generalized aminoaciduria wað detected. Generalized osteoporosis, renal tubulae dysfunction resembling that seen in the Fanconi syndrome accompanied by metabolic acidosis and hypopotassaemis were diagnosed. Subsequently, homocystine was identio fied in the urinary chromatogram; cystine was no present. Steatorrhoea developed during his stay hospital.

At laparotomy, $100 \mathrm{ml}$. of clear, pale ascitic fluid an $\bar{\Phi}$ moderate retroperitoneal oedema was noted. Thrombus was not detected in the inferior vena cava or othe? abdominal vessels. The stomach and duodenum appeare normal; the small bowel was pale and peristalsis wasnormal. Lymph nodes were not enlarged. The gafs bladder, spleen, pancreas, and adrenals appeared normal The kidneys were rather larger than normal but nog otherwise noteworthy. The liver was markedly enlarged, soft, yellowish-pink with a smooth surface and a rounded edge.

After discharge from hospital the patient died from a年 unknown cause at the age of 8 years. A necropsy was noop obtained.

In a surgical biopsy of liver, a marked degree of fatto change was seen. Fatty change extended from the centre of each lobule almost as far as the portal tracts and it was only round the portal tracts that the liver cells had the normal form. Some of the fatty cells were grossly ero larged. Collections of polymorphs and Mallory's hyaling were not present. There was no fibrosis. No features of 
special note were seen in the portal tracts or in their vessels.

\section{CASE 4}

A girl, P.B., aged $6 \frac{1}{2}$ years, who was mentally retarded, was diagnosed as a case of homocystinuria by urinary chromatography. Her parents are healthy and unrelated; there is a history of two miscarriages. A sister of the patient then aged 4 has the mental and physical characteristics of the propositus and is also excreting homocystine. A brother then aged 2 is healthy and intelligent.

The patient presented a retarded appearance, a high malar flush, and bilateral ectopia lentis, pes cavus, and genu valgum were present. No arachnodactyly or other skeletal abnormality was noted. The liver was enlarged.

The findings from a needle biopsy of the liver are the same as in the liver biopsy of case 3 .

Biochemical observations on this case are recorded by Carson et al. (1963).

\section{DISCUSSION}

Homocystine is probably derived from methionine, the essential sulphur-containing amino-acid in the diet. In normal metabolism methionine is broken down through homocysteine and cystathionine to cysteine and cystine and this pathway is of general biological importance. Classical cystinuria is well known as a renal abnormality, and cystathioninuria with mental defect has been described by Harris, Penrose, and Thomas (1959) but it is not clear how they may be related to the present condition. Homocystine, on the other hand, has not yet been detected in the normal pathway and is not a recognized constituent of normal urine. The metabolism of methionine in homocystinuria was investigated by Carson et al. (1963). Methionine loading was employed, and the results were of a different kind from those found in cystathioninuria. Although the rate of disappearance of methionine from the blood was less than in normal controls, the amount of homocystine excreted was insufficient to account for the methionine metabolized. It thus appears that only a portion of the methionine is metabolized abnormally. It was concluded that in homocystinuria there is a defect in methionine metabolism although the defect was not defined precisely. An inability of the renal tubules to reabsorb methionine in the normal way was also demonstrated and this suggests a generalized transport defect in the handling of this amino-acid.

The relationship of sulphydryl-containing aminoacids to liver function has been studied extensively, and particularly the effects of dietary deficiency. In mice homocystine largely replaces methionine in the diet provided choline, folic acid and vitamin $\mathbf{B}_{12}$ are also given (Sauberlich, 1959). When choline is omitted, liver fat is greatly increased and growth is reduced. Feeding of homocystine appears deleterious in itself, for even with the addition of methionine to the diet, growth is not entirely normal. A comparable effect is evident in our cases of homocystinuria, for a marked degree of fatty change was found in liver biopsies from cases 3 and 4 and less marked fatty change at necropsy in case 1 (Fig. 3) where the findings may have been modified by the vascular changes.

Because sulphydryl-containing amino-acids are intricately involved in many vital processes, disorders of their metabolism might be expected to produce effects correspondingly widespread. The present study, however, suggests that cerebral function and the processes of thrombosis and of fibrogenesis are particularly vulnerable.

\section{CEREBRAL FUNCTION}

Mental defect appears to be an inevitable element of homocystinuria, for the condition has been detected only in mentally retarded individuals. Further surveys are needed to confirm the association, but it is not unexpected in view of the general occurrence of mental defect in inborn errors of amino-acid metabolism. Because of the coincident finding of Marfan's syndrome in some patients with homocystinuria, it is noteworthy that McKusick (1960) does not consider mental retardation a component of Marfan's syndrome. A morphological basis for the general occurrence of mental defect was not established in our material. The brain was studied in case 1 only, where the left cerebral hemisphere was extensively damaged by healed, venous infarctions (Figs. 2 and 4).

\section{THROMBOSIS}

Indications of previous venous thrombosis were found in the superior sagittal sinus in case 1 and are noteworthy in the histories of other cases. In a brother of case 1 , who is excreting homocystine, thrombosis of the inferior vena cava has been diagnosed. Case 3 was admitted to hospital with recurrent thromboses of the lower limbs. In the material studied a venous lesion has not been encountered that might predispose to thrombosis. Arterial thrombus was found in case 1, not only in a renal artery as the cause of death, but also partly organized in the aorta. Thrombi were found in other arteries of abnormal structure (Fig. 6). Other vascular lesions may possibly have resulted from mural thrombosis, but this is uncertain, for iron pigment was not found in the intimal pads and ridges in the aorta (Figs. 1, 5, 8, and 11) and muscular arteries in case 1 , or in the renal artery branches in 
case 2 (Figs. 11 and 12). These intimal thickenings may perhaps be related to underlying defects of the medial coat but the frequency of both venous and arterial thrombosis suggests the operation of a haemic factor, though none of the investigations in the present cases showed what this might be. Since defective platelet function might cause both mural and occlusive thrombi, it is of interest that Koppel (1960) found that incubation with sulphydryl inhibitors caused an increased breakdown of platelets. He suggested a direct relationship between platelet sulphydryl groups and platelet structure. The experiments of Fantl, Ebbels, and Nelson (1951) lead to similar conclusions. Unpublished preliminary results of platelet investigations on the affected sister of case 4 indicate that platelet stickiness is abnormal in homocystinuria (Macdonald, personal communication).

\section{ASSOCIATION WITH MARFAN'S SYNDROME}

Cases 2 and 3 were overt examples of Marfan's syndrome (Fig. 10) with no family history such as is encountered in Marfan's disease. Several elements of the syndrome are recognizable in all the cases, however, pes cavus, kyphoscoliosis, and ectopia lentis in particular. Ectopia lentis is not confined to Marfan's syndrome but in $70 \%$ of cases it is a component of that condition (McKusick, 1960). McKusick comments that 'the skeletal manifestations of the Marfan syndrome may be so unconvincing in many cases that the presence of ectopia lentis in a patient with cardiovascular signs consistent with Marfan's syndrome should lead one to suspect the diagnosis even though the skeletal changes per se cannot be considered pathognomonic'. These remarks have a bearing on case 1 in which arachnodactyly was not present. Abnormal bosses in the skull were discovered only at necropsy which also displayed extensive vascular changes and some of them were of the type found in Marfan's syndrome. The aortic valve ring was dilated and its diameter was greater than that of the pulmonary valve. The aorta was dilated down to the renal arteries and metachromatically-staining medial degeneration was found; the arch was the portion most affected. It is thus permissible to classify case 1 as of Marfan's syndrome. Coarctation of the aorta has been described in Marfan's syndrome and may be due there to the same connective tissue anomaly as dissecting aneurysm (McKusick, 1960). In case 1, the bands round the aorta at the level of the left subclavian artery were built up of fibrous tissue in the intima and did not take the form of an indrawing of the media as seen in true coarctation. Metachromatic medial degeneration extended into the medial coats of some large muscular arteries, including the left $\frac{\mathbb{H}^{*}}{5}$ renal (Fig. 6) and splenic arteries, and small aneurysms had formed in the superior mesenteric $\overrightarrow{\vec{F}}$ artery and left renal arteries. Similar arterial $\stackrel{?}{+}$ involvement has been recorded in other cases of $?$ Marfan's syndrome (Austin and Schaefer, 1957; $\frac{\sigma}{\bar{c}}$ Roark, 1959; McKusick, 1960). Marfan's syndrome $\frac{\text { }}{\widetilde{D}}$ is an uncommon condition. Lynas (1958) reported $\stackrel{\mathbb{Q}}{\square}$ only 20 cases living in a population of $1,370,921$ in Northern Ireland and its occurrence in three out of $\overrightarrow{0}$ our four cases must be taken into account in any $\vec{A}$ discussion of homocystinuria.

Medial degeneration with muscular and elastic $\frac{\text { ? }}{2}$ vascular defects are characteristic of Marfan's? syndrome, but the intimal pads and ridges described $\vec{v}$ in cases 1 and 2 (Figs. 1, 5, 8, and 11) are not. We? have not found similar lesions in a study of two t unrelated cases of Marfan's syndrome used as ${ }_{0}^{\gamma}$ control material, but Roark (1959) has described them as a unique finding in this condition. The $\vec{C}$ possible relationship of these pads to mural thrombosis has been mentioned above. Since they are asymmetrical they may be distinguished fromo any changes secondary to the persistent hypertension in case 2 (Fig. 11). Hypertension is rare in Marfan's syndrome (McKusick, 1960). It was recorded only in this one of our cases and it was ato first thought to be the result of a previous attack of nephritis (Loughridge, 1959) but no evidence of nephritis was found when the ectopic left kidney was $\vec{\circ}$ removed surgically. Hypertension in this boy is 3 probably the result of the renal arterial lesions and it is noteworthy that the juxtaglomerular apparatus was unusually prominent in the resected kidney. The patient's blood pressure reverted to normal after the operation and it has not been possible to study him further.

The finding of cases of the new aminoacidurias with features of Marfan's syndrome suggests that some older cases of homocystinuria may have been? described in papers on that syndrome. In a famils with Marfan's syndrome, Lehmann (1960) reported: urinary excretion of 'cystine' and lysine in twor sibs, one of whom showed Marfan features. Since homocystine may be unwittingly mistaken fon cystine, Lehmann's report is of particular interest ${ }_{\sigma}^{\omega}$ Lysine was present in the urine of our case 3 but only as part of a generalized aminoaciduria. Parke Weber (1933) described a man of 19 with sub luxation of both lenses, scoliosis and thrombosis of the internal iliac veins or inferior vena cava similar ocular and thoracic deformities were presen $\bar{\Phi}$ in a younger sister. Parkes Weber suggested that the्ष condition was allied to arachnodactyly. The parent? were not affected. The intelligence of these patients was not indicated. The resemblance of such cases to 
homocystinuria is rather strong, but in most instances comparisons can only be speculative. In future, however, urine should be examined for homocystine in cases where Marfan's syndrome occurs with feeblemindedness (Stewart, 1939; Wahl and Schachter, 1940; Gibson 1956).

Weve (1931) first clearly demonstrated the genetic nature of Marfan's syndrome. His characterization of Marfan's syndrome as a congenital mesodermal dystrophy remains an acceptable limited description of Marfan's disease with a dominant type of familial transmission. None of the individual lesions of Marfan's disease, however, can be regarded as specific for the condition, because some of them occur in isolated form in other states, and because the skeletal changes, at least, may be modified by other genetic determinants. When the full diagnostic triad of skeletal, cardiac, and ocular manifestations is present without a family history, the term Marfan's syndrome is appropriate. Three of our four cases may be classified in this way. It appears that in a significant number of cases of homocystinuria the development or maintenance of certain structures may be altered in just the same way as in Marfan's disease. The two underlying conditions are of course distinct. Homocystinuria is probably transmitted as an autosomal recessive while Marfan's disease is transmitted as an autosomal dominant. Under appropriate circumstances, however, both conditions may manifest themselves through the same 'final common pathway' of structural damage which may be shared by other agents. Probably this pathway lies through processes whereby fibrocytes form collagen and elastic fibres, but the role of sulphur in this metabolic pathway has not yet been established. Studies of mucopolysaccharide metabolism in Marfan's syndrome have shown that large amounts of keratosulphate are present in the bones and aorta (Meyer, Hoffman, and Linker, 1958) and that serum mucoprotein levels are low (Bacchus, 1958). Urinary estimations of hydroxyproline have yielded conflicting results (Sjoerdsma, Davidson, Udenfriend, and Mitoma, 1958; Lehmann 1960).

Another approach is suggested by the reports of scoliosis and dissecting aneurysms in young rats fed lathyrus odoratus (Ponseti and Baird, 1952; McKay, Lalich, Schilling, and Strong, 1954) and by the similar effects of some derivatives on the chick embryo (Gross, Levene, and Orloff, 1960; Levene, $1958,1961)$. The experiments of Levene and Gross (1959) indicated an alteration in the molecular aggregation of collagen when $\beta$-aminopropionitrile was administered to chick embryos. It is in keeping with modern thought to suppose that an inborn error of metabolism might alter fibrogenesis through enzymatic or other effects in a similar way. Possibly accumulations of homocystine alone do not determine these effects; at least Levene (1961) found homocystine and other SH-containing compounds without lathyrogenic activity in the chick embryo system. Some less direct effect of the disordered methionine metabolism of our patients is perhaps responsible. At all events the association in a number of ways of the specific aminoaciduria with Marfan's syndrome suggests that homocystinuria may be related to abnormalities of connective tissue. Experimental lathyrism offers a method by which this association may be explored, not only in relation to Marfan's syndrome but also in relation to general problems of fibrogenesis.

We are indebted to Dr. T. W. H. Weir, Medical Superintendent, Special Care Centre, Muckamore, Co. Antrim, for his continued cooperation. He and other medical colleagues have been most willing to permit us access to patients under their care. We have had helpful discussions with Professor C. E. Dent of University College Hospital, London, and we are indebted to him for introducing us to Dr. Lawson Macdonald who carried out studies on the platelet abnormalities.

We are grateful to Mr. D. McA. Mehaffey and Mr. R. Woods for the photographs.

One of us (N.A.J.C.) acknowledges with gratitude a research grant from the Hospital Committee of the Royal Belfast Hospital for Sick Children.

\section{REFERENCES}

Austin, M. G., and Schaefer, R. F. (1957). Arch. Path., 64, 205. Bacchus, H. (1958). Amer. J. Med., 25, 744.

Carson, N. A. J. (1963). M.D. Thesis, Queen's University, Belfast. , Cusworth, D. C., Dent, C. E., Field, C. M. B., Neill, D. W., and Westall, R. G. (1963). Arch. Dis. Childh., 38, 425. and Neill, D. W. (1962). Ibid., 37, 505.

Fantl, P., Ebbels, L., and Nelson, J. F. (1951). Brit. J. exp. Path., 32, 538.

Gibson, R. (1956). Canad. med. Ass. J., 75, 501.

Gross, J., Levene, C. I., and Orloff, S. (1960). Proc. Soc. exp. Biol. (N.Y.), 105, 148.

Harris, H., Penrose, L. S., and Thomas, D. H. H. (1959). Ann. hum. Genet., 23, 442.

Koppel, J. L. (1960). In International Symposium: Blood Platelets, Henry Ford Hospital, Detroit, edited by S. A. Johnson et al., p. 423. Little, Brown, Boston.

Lehmann, O. (1960). Acta paediat. (Uppsala), 49, 540.

Levene, C. I. (1958). Fed. Proc., 17, 445.

(1961). J. exp. Med., 114, 295.

- , and Gross, J. (1959). Ibid., 110, 771.

Loughridge, L. W. (1959). Quart. J. Med., 28, 531.

Lynas, M. A. (1958). Ann. hum. Genet., 22, 289.

MacDonald, L. (1963). Personal communication.

McKay, G. F., Lalich, J. J., Schilling, E. D., and Strong, F. M. (1954). Arch. Biochem., 52, 313.

McKusick, V. A. (1960). Heritable Disorders of Connective Tissue. 2nd ed., p. 103. Mosby, St. Louis.

Meyer, K., Hoffman, P., and Linker, A. (1958). Science, 128, 896. Ponseti, I. V., and Baird, W. A. (1952). Amer. J. Path., 28, 1059.

Roark, J. W. (1959). Arch. intern. Med., 103, 123.

Sauberlich, H. E. (1959). J. Nutr., 68, 141.

Sjoerdsma, A., Davidson, J. D., Udenfriend, S., and Mitoma, C. (1958). Lancet, 2, 994.

Stewart, R. M. (1939). Arch. Dis. Childh., 14, 64.

Wahl, M., and Schachter, M. (1940). Arch. Méd. Enf., 43, 37.

Weber, F. P. (1933). Lancet, 2, 1472.

Weve, H. (1931). Arch. Augenheilk., 104, 1. 